ARTICLE

\title{
Efficient hydrogen evolution by ternary molybdenum sulfoselenide particles on self-standing porous nickel diselenide foam
}

Haiqing Zhou ${ }^{1}$, Fang Yu ${ }^{1}$ Y Yufeng Huang ${ }^{2}$, Jingying Sun ${ }^{1}$, Zhuan Zhu ${ }^{3}$, Robert J. Nielsen², Ran He ${ }^{1}$ Jiming Bao ${ }^{3}$, William A. Goddard ${ } \mathrm{I}^{2}$, Shuo Chen ${ }^{1} \&$ Zhifeng Ren ${ }^{1}$

With the massive consumption of fossil fuels and its detrimental impact on the environment, methods of generating clean power are urgent. Hydrogen is an ideal carrier for renewable energy; however, hydrogen generation is inefficient because of the lack of robust catalysts that are substantially cheaper than platinum. Therefore, robust and durable earth-abundant and cost-effective catalysts are desirable for hydrogen generation from water splitting via hydrogen evolution reaction. Here we report an active and durable earth-abundant transition metal dichalcogenide-based hybrid catalyst that exhibits high hydrogen evolution activity approaching the state-of-the-art platinum catalysts, and superior to those of most transition metal dichalcogenides (molybdenum sulfide, cobalt diselenide and so on). Our material is fabricated by growing ternary molybdenum sulfoselenide particles on self-standing porous nickel diselenide foam. This advance provides a different pathway to design cheap, efficient and sizable hydrogen-evolving electrode by simultaneously tuning the number of catalytic edge sites, porosity, heteroatom doping and electrical conductivity.

\footnotetext{
${ }^{1}$ Department of Physics and TcSUH, University of Houston, Houston, Texas 77204, USA. ${ }^{2}$ Materials and Process Simulation Center (139-74), California Institute of Technology, Pasadena, California 91125, USA. ${ }^{3}$ Department of Electrical and Computer Engineering, University of Houston, Houston, Texas 77204 , USA. Correspondence and requests for materials should be addressed to S.C. (email: schen34@uh.edu) or to Z.R. (email: zren@uh.edu).
} 
$\mathrm{T}$ he large consumption of fossil fuels and its impact on the environment make it urgent to develop environmentally friendly and renewable energy sources. Hydrogen $\left(\mathrm{H}_{2}\right)$ is an attractive and promising energy carrier because of its high energy density and no pollution gas emission ${ }^{1,2}$. One direct and effective route to generate $\mathrm{H}_{2}$ is based on electrocatalytic hydrogen evolution reaction (HER) from water splitting, in which an efficient catalyst is required to ensure the energy efficiency ${ }^{3-5}$. Platinum (Pt)-based noble metals are by far the most active catalysts; however, they are not suitable for large-scale applications because of the high cost and scarcity of Pt on earth ${ }^{6}$. Thus, we aim to identify alternative electrocatalysts based on earth-abundant and cost-effective elements ${ }^{7,8}$. Until now, various classes of earth-abundant transition metal compounds are confirmed to be promising candidates ${ }^{9,10}$, such as metal sulfides, selenides, phosphides, carbides and the composites. However, thus far most of the catalysts exhibit inferior efficiency to Pt, while many involve complicated preparation methods and multiple steps that increase costs. Great progress has been obtained for HER based on layered transition metal dichalcogenides (LTMDs) such as molybdenum disulfide $\left(\mathrm{MoS}_{2}\right)$ either in the form of crystalline or amorphous states ${ }^{9-12}$, and even in molecular mimics ${ }^{13}$; however, it remains a challenge to get catalytic performance comparable to that of $\mathrm{Pt}$, which is probably due to the low density and reactivity of active sites, poor electrical transport and inefficient electrical contact to the catalyst ${ }^{14-16}$.

On the basis of the above results, fabricating $\mathrm{MoS}_{2}$ or its derivatives into hybrids or composites might be an interesting strategy to promote the catalytic performance ${ }^{17}$. Currently, carbon-based materials are generally used as the catalyst support because of their high surface area and good conductivity ${ }^{77,18}$. However, complex catalyst synthesis procedures are typically required. As an alternative, arranging the catalysts into doublegyroid structures with numerous nanopores might lead to improved HER activity because of preferential exposure of catalytic active edges rather than the inactive basal planes ${ }^{19}$. This approach reminds us of the necessity to make three-dimensional (3D) catalysts with high surface area loaded on porous supports, fast proton transfer and greater contact areas with reactants during the catalytic process. Meanwhile, the bottleneck of the double-gyroid structures is the intrinsically poor conductivity of the catalysts. Thus, even though $\mathrm{MoS}_{2}$ is established as an effective HER catalyst since 2005 (ref. 11), it is still difficult to obtain satisfactory catalysts in low costs on par with the current $\mathrm{Pt}$ catalysts. In the light of these challenges, we conclude that the best strategy is to improve the dispersion and electrical conductivity of these catalysts on the supports and to expose a large number of active edge sites. Furthermore, we consider that arranging two different materials into hybrids might lead to synergistic effects ${ }^{20,21}$ that utilize the best properties of each component.

In this work, we propose a strategy of growing ternary molybdenum sulfoselenide $\mathrm{MoS}_{2(1-x)} \mathrm{Se}_{2 x}$ particles with vertically aligned layers on a 3D porous HER-active conductive nickel diselenide $\left(\mathrm{NiSe}_{2}\right)$ scaffold, which takes advantage of the merits of highly conductive support, double-gyroid structures $(3 \mathrm{D}$, porous and lots of exposed edge sites) and synergistic effects between two different catalysts. Indeed, we measure excellent HER performance for this hybrid catalyst that is superior to most reported transition metal dichalcogenides $\left(\mathrm{MoS}_{2}\right.$, cobalt diselenide $\mathrm{CoSe}_{2}$ and so on).

\section{Results}

Preparation of 3D porous hybrid electrocatalyst. To the best of our knowledge, the majority of HER catalysts reported thus far are based on nanostructures (nanoparticles, nanosheets and so on); thus, binder polymers (for example, nafion solution) are necessary to fasten the catalysts on the conducting substrates such as glassy carbon electrodes, which somewhat increases the cost. This problem can be avoided by growing the active catalysts directly on self-standing conducting skeletons as the current collectors $^{22-24}$. The key challenge is to find a suitable 3D supports with high surface area, high porosity and good conductivity. Graphene or carbon nanotube is not feasible because of their high costs. Instead, nickel (Ni) foam is suitable because of its low price, commercial availability and 3D skeleton structure ${ }^{25}$ (Fig. 1a). However, Ni foam is not stable in acidic electrolytes because of corrosion. Interestingly, our previous work shows that direct selenization in Ar atmosphere can convert $\mathrm{Ni}$ foam to porous $\mathrm{NiSe}_{2}$ foam (Fig. 1b,c, Supplementary Fig. 1 and Supplementary Note 1 ) that is HER-active and very stable in acid ${ }^{26}$. We find that numerous additional pores are generated in the $\mathrm{NiSe}_{2}$, which provides preferential sites for growing LTMD catalysts with high-density active edges ${ }^{27}$. Thus, we propose using $3 \mathrm{D}$ porous $\mathrm{NiSe}_{2}$ foam as a conductive skeleton to load ternary $\mathrm{MoS}_{2(1-x)} \mathrm{Se}_{2 x}$ catalysts (Supplementary Note 2), thereby utilizing the excellent electrical conductivity, porous structures and high surface area of the $\mathrm{NiSe}_{2}$ foam (Fig. 1d,e). Indeed, scanning electron microscopy (SEM; Fig. 1d,e and Supplementary Note 3) images clearly show the uniform distribution of small ternary particles on porous $\mathrm{NiSe}_{2}$ foam, which is important for the electrocatalytic performance of LMDT catalysts.

Structural characterizations of the electrocatalyst. The chemical composition of the as-grown particles was examined using high-resolution transmission electron microscopy (TEM), X-ray photoelectron spectroscopy (XPS), Raman spectroscopy and energy-dispersive X-ray spectroscopy. TEM images (Fig. 2a,b and Supplementary Fig. 2) clearly resolve a large amount of vertically aligned $\mathrm{MoS}_{2(1-x)} \mathrm{Se}_{2 x}$ layers, suggesting that many active edge sites are exposed at the surface of $\mathrm{MoS}_{2(1-x)} \mathrm{Se}_{2 x}$ particles. It is reasonable since the porous structure of $\mathrm{NiSe}_{2}$ foam with high surface area is favourable for the growth of layered materials with vertically aligned layers ${ }^{27}$. Meanwhile, XPS spectra in the hybrid reveal the presence of $\mathrm{Ni}, \mathrm{Mo}, \mathrm{S}$ and Se elements (Fig. 2c-e). However, since the $\mathrm{Se}$ in $\mathrm{NiSe}_{2}$ foam has a similar state to that in $\mathrm{MoS}_{2(1-x)} \mathrm{Se}_{2 x}$, it is difficult to demonstrate the selenization of $\mathrm{MoS}_{2}$ on porous $\mathrm{NiSe}_{2}$ foam. Instead, to confirm the chemical composition of the molybdenum compound, we put a precursordecorated $\mathrm{Si}$ substrate underlying the $\mathrm{NiSe}_{2}$ foam during the second selenization. It is clear that the $\left(\mathrm{NH}_{4}\right)_{2} \mathrm{MoS}_{4}$ precursor has been converted to a distinctive ternary alloy phase at $500{ }^{\circ} \mathrm{C}$ from the prominent Mo, S and Se signals in the XPS spectra ${ }^{28}$ (Fig. 2c-e). Especially in the Raman spectra (Fig. 2f), in comparison with pure $\mathrm{MoS}_{2}$ that exhibits two prominent peaks at $380 \mathrm{~cm}^{-1}\left(E_{1 \mathrm{~g}}\right)$ and $406 \mathrm{~cm}^{-1}\left(A_{2 \mathrm{~g}}\right)$, there is another obvious peak located at $264 \mathrm{~cm}^{-1}$ for the samples with a ternary phase, which can be ascribed to the $A_{1 \mathrm{~g}}$ mode of the Mo-Se bond ${ }^{29}$. Compared with the Raman mode of the bulk $\mathrm{MoSe}_{2}$ crystals $\left(\sim 242 \mathrm{~cm}^{-1}\right)$, the blueshifts of this peak to $264 \mathrm{~cm}^{-1}$ suggest a ternary $\mathrm{MoS}_{2(1-x)} \mathrm{Se}_{2 x}$ compound rather than a mixture of two solid phases. This Raman feature is also observed from the ternary phase grown on porous $\mathrm{NiSe}_{2}$ foam, which is consistent with previously reported results on ternary $\mathrm{MoS}_{2(1-x)} \mathrm{Se}_{2 x}$ single crystals $^{29}$. By comparing the relative peak intensity between 264 and $380 \mathrm{~cm}^{-1}$, we estimate that the atomic ratio between $S$ and Se is $\sim 1$, which is further supported by the energy-dispersive $\mathrm{X}$-ray spectroscopy analysis (Supplementary Fig. 3).

Hydrogen evolution catalysis. Considering the metallic and porous feature in the $\mathrm{NiSe}_{2}$ foam, and the good dispersion and preferential layer orientation of ternary $\mathrm{MoS}_{2(1-x)} \mathrm{Se}_{2 x}$ 


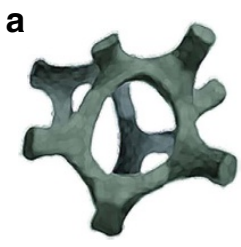

3D Ni foam

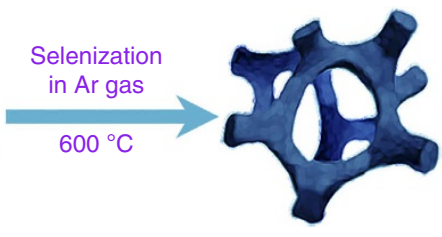

3D porous

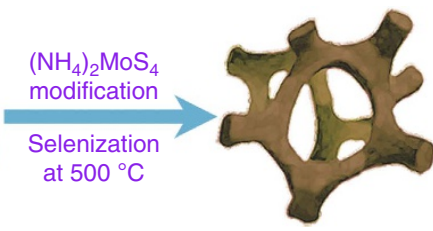

3D porous MoSSe/NiSe ${ }_{2}$ foam
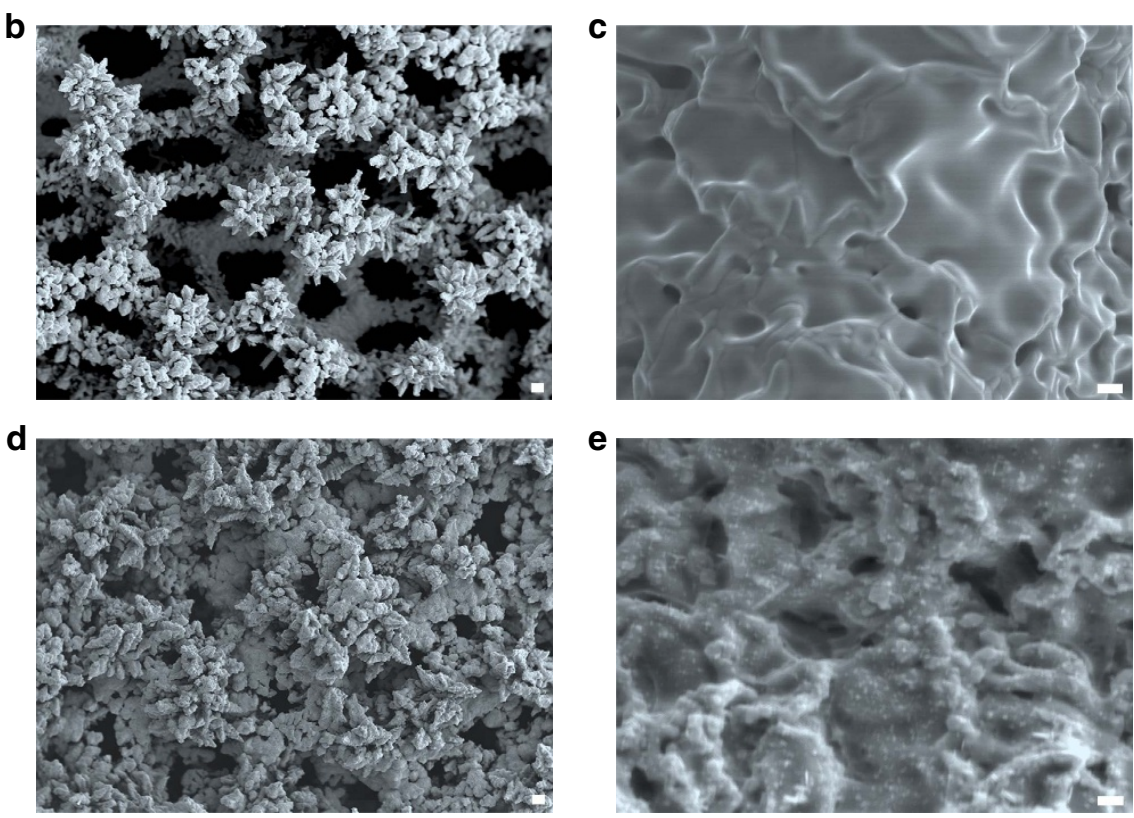

Figure 1 | The schematic diagram and morphology characterizations. (a) The procedures for growing ternary $\mathrm{MoS}_{2(1-x)} \mathrm{Se}_{2 x}$ particles on porous $\mathrm{NiSe}_{2}$ foam. (b,c) Typical SEM images showing the surface roughness of the $\mathrm{NiSe}_{2}$ foam grown at $600^{\circ} \mathrm{C}$ from commercial Ni foam. (d,e) Typical SEM images showing the morphologies of ternary $\mathrm{MoS}_{2(1-x)} \mathrm{Se}_{2 x}$ particles distributed on porous $\mathrm{NiSe}_{2}$ foam grown at $500^{\circ} \mathrm{C}$. $(\mathbf{b}, \mathbf{d}) \mathrm{Scale}$ bar, $50 \mu \mathrm{m}$. (c,e) Scale bar, $1 \mu \mathrm{m}$.

particles, it is expected that this 3D architecture should have outstanding HER activity, such as low overpotentials, low Tafel slopes and large exchange current densities. To evaluate the catalytic performance of these ternary $\mathrm{MoS}_{2(1-x)} \mathrm{Se}_{2 x}$ particles on 3D porous $\mathrm{NiSe}_{2}$ foam, we performed detailed electrocatalytic measurements via a standard three-electrode set-up in a $0.5 \mathrm{M}$ $\mathrm{H}_{2} \mathrm{SO}_{4}$ electrolyte de-aerated with high-purity $\mathrm{N}_{2}$. The loading of $\mathrm{MoS}_{2(1-x)} \mathrm{Se}_{2 x}$ catalysts is $\sim 4.5 \mathrm{mg} \mathrm{cm}^{-2}$. Figure 3a shows that the self-standing porous hybrid catalyst can afford a geometric current density of $-10 \mathrm{~mA} \mathrm{~cm}^{-2}$ at a very low overpotential of $-69 \mathrm{mV}$ for the ternary $\mathrm{MoS}_{2(1-x)} \mathrm{Se}_{2 x} / \mathrm{NiSe}_{2}$ hybrid electrode (Supplementary Table 1). In contrast, for binary $\mathrm{MoS}_{2}$ on $\mathrm{NiSe}_{2}$ foam and pure $\mathrm{NiSe}_{2}$ foam, overpotentials of -118 and $-153 \mathrm{mV}$ are needed to achieve $-10 \mathrm{~mA} \mathrm{~cm}^{-2}$, respectively. The catalytic overpotential $(-69 \mathrm{mV})$ of the $\mathrm{MoS}_{2(1-x)} \mathrm{Se}_{2 x} /$ $\mathrm{NiSe}_{2}$ hybrid is also much lower than those of the best catalysts thus far based on LTMDs $\operatorname{MoS}_{2}(-110 \mathrm{mV})^{18}$, $\mathrm{WS}_{2}(-142 \mathrm{mV})^{15}$ and $\mathrm{WS}_{2(1-x)} \mathrm{Se}_{2 x}(-170 \mathrm{mV})^{30}$, and firstrow transition metal dichalcogenides $\mathrm{CoSe}_{2}(-139 \mathrm{mV})^{24}, \mathrm{NiSe}_{2}$ $(-136 \mathrm{mV})^{26}$ and $\operatorname{CoS}_{2}(-142 \mathrm{mV})^{31}$, suggesting that our ternary $\mathrm{MoS}_{2(1-x)} \mathrm{Se}_{2 x}$ particles/ $\mathrm{NiSe}_{2}$ foam hybrid is an outstanding HER catalyst. Meanwhile, a Tafel slope, which is an inherent property of the catalyst, can be obtained by extracting the slopes from the linear regions in Tafel plots (Fig. 3b). We find that the ternary electrode possesses a smaller Tafel slope of $42.1 \mathrm{mV}$ per decade than that of binary $\mathrm{MoS}_{2}$ on $\mathrm{NiSe}_{2}$ foam ( $58.5 \mathrm{mV}$ per decade) and pure $\mathrm{NiSe}_{2}$ foam $(46.4 \mathrm{mV}$ per decade). In addition, our hybrid catalyst leads to a Tafel slope much lower than many previously reported cheap and efficient HER catalysts in the same electrolyte (Supplementary Table 2). More interestingly, based on the intercept of the linear region of the Tafel plots, the exchange current densities $\left(j_{0}\right.$,geometrical $)$ at the thermodynamic redox potential $(\eta=0)$ can be calculated to be $299.4 \mu \mathrm{A} \mathrm{cm}^{-2}$ for the ternary-phase hybrid catalysts. This exchange current density is one to two orders of magnitude larger than those of well-known LTMDs $\mathrm{MoS}_{2}$ and $\mathrm{WS}_{2}$, or first-row transition metal dichalcogenides $\mathrm{CoSe}_{2}$ and $\mathrm{CoS}_{2}$ catalysts (Supplementary Table 2). Thus, considering the small overpotential $\left(-69 \mathrm{mV}\right.$ to reach $\left.10 \mathrm{~mA} \mathrm{~cm}^{-2}\right)$, low Tafel slope $(\sim 42.1 \mathrm{mV}$ per decade) and large exchange current density $\left(\sim 299.4 \mu \mathrm{A} \mathrm{cm}^{-2}\right)$, it is worth pointing out that the catalytic performance of our as-prepared catalyst is superior to most of the $\mathrm{MoS}_{2}$-based catalysts.

Aside from a stringent requirement for high HER activity, stability is another important criterion in evaluating the performance of an electrocatalyst. In our experiment, a long-term cyclic voltammetry $(\mathrm{CV})$ test between -0.20 and $0.07 \mathrm{~V}$ versus RHE shows no significant degradation of cathodic current densities for the hybrid catalyst after 1,000 cycles (Fig. 3c). Particularly, the cathodic current density for the hybrid catalyst remains stable and exhibits no obvious degradation for electrolysis at a given potential $(-69$ or $-121 \mathrm{mV})$ for over a long period ( $>16 \mathrm{~h}$; Fig. $3 \mathrm{~d}$ ), suggesting the potential use of this catalyst over a long time in an electrochemical process. Even after long-term stability and cyclability tests, the catalytic performance of this hybrid catalyst still shows no degradation compared with 
a

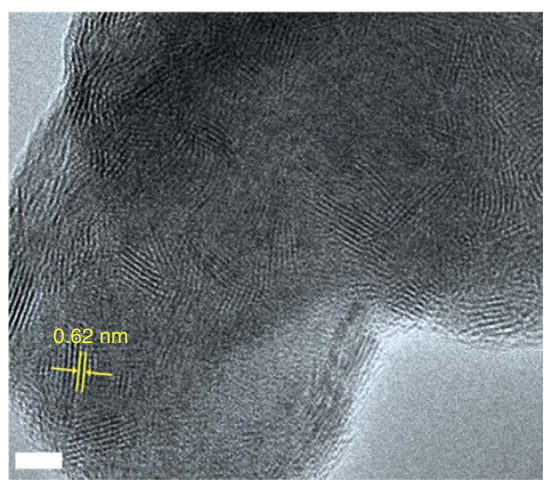

d

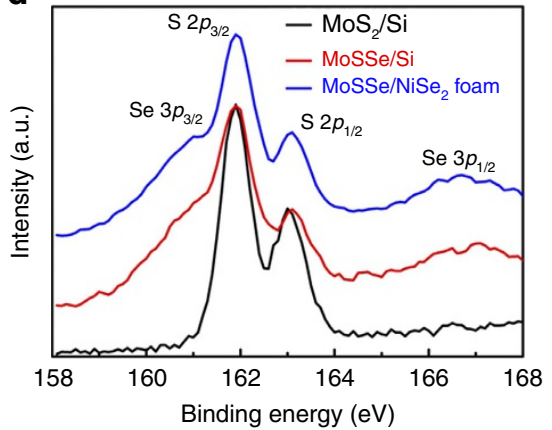

b

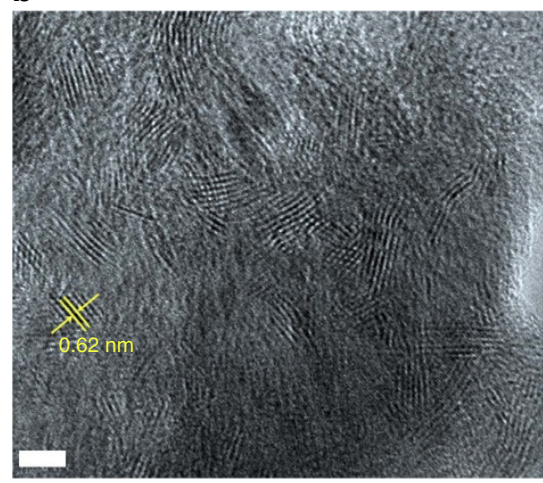

e

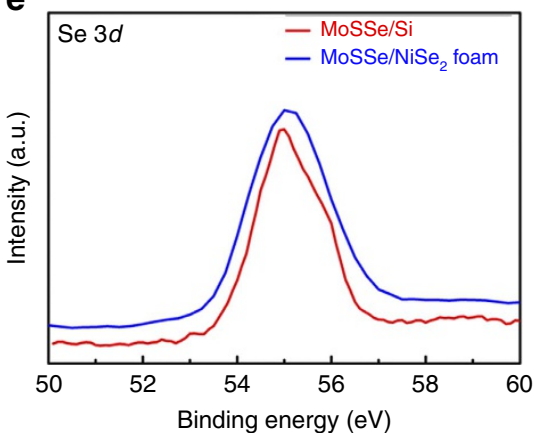

c
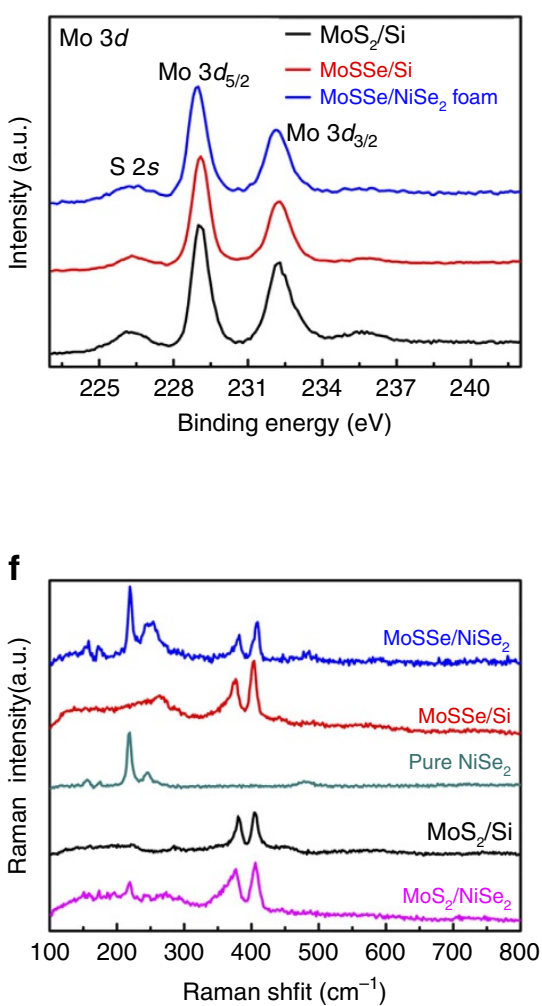

Figure 2 | Characterization of the ternary $\mathbf{M o S}_{\mathbf{2 ( 1 - x )}} \mathbf{S e}_{\mathbf{2 x}} / \mathbf{N i S e}_{\mathbf{2}}$ foam hybrid catalysts. (a,b) TEM images showing the vertical layer orientation of $\mathrm{MoS}_{2(1-x)} \mathrm{Se}_{2 x}$ particles grown on different regions of porous NiSe 2 foam. Scale bar, $5 \mathrm{~nm}$. (c-e) Detailed XPS analysis of the Mo $3 d, \mathrm{~S} 2 p$ and Se $3 d$ spectra in different samples, such as binary $\mathrm{MoS}_{2}$ particles on $\mathrm{Si}, \mathrm{MoS}_{2(1-x)} \mathrm{Se}_{2 x}$ particles on $\mathrm{Si}$ and $\mathrm{MoS}_{2(1-x)} \mathrm{Se}_{2 x}$ particles on porous NiSe 2 foam. (f) Raman spectra measured on different samples.

its initial state (Fig. 3c). In addition, the Faradaic efficiency for hydrogen evolution of this hybrid catalyst was evaluated (Supplementary Note 4). The efficiency is determined to be nearly 100\% during 60 min of electrolysis (Supplementary Fig. 4).

To elucidate the origin of the differences in the overall catalytic performance among different catalysts, a simple CV method $^{15,20,24}$ was utilized to measure the corresponding electrochemical double-layer capacitances $\left(C_{\mathrm{dl}}\right)$ for evaluation of the electrochemically effective surface areas (Supplementary Fig. 5). Taking consideration of the direct proportion between the effective surface area and double-layer capacitance, we just need to compare the capacitance values $C_{\mathrm{dl}}$. By plotting the positive and negative current density differences $\left(\Delta j=j_{\mathrm{a}}-j_{\mathrm{c}}\right)$ at a given potential $(0.15 \mathrm{~V}$ versus $\mathrm{RHE})$ against the $\mathrm{CV}$ scan rates, we can directly get the $C_{\mathrm{dl}}$, which is equal to half the value of the linear slopes of the fitted lines in the plots. As shown in Fig. 3e, the $\mathrm{MoS}_{2(1-x)} \mathrm{Se}_{2 x} / \mathrm{NiSe}_{2}$ hybrid electrode exhibits a $C_{\mathrm{dl}}$ value of $319.15 \mathrm{mF} \mathrm{cm}^{-2}$, which is one order of magnitude larger than that of the pure $\mathrm{MoS}_{2} / \mathrm{NiSe}_{2}$ foam $\left(30.88 \mathrm{mF} \mathrm{cm}^{-2}\right)$, and $\sim 43$ times larger than that of pure $\mathrm{NiSe}_{2}$ foam $\left(7.48 \mathrm{mF} \mathrm{cm}^{-2}\right)$, demonstrating the proliferation of active sites in the porous hybrid catalyst, which accordingly results in the improved catalytic performance. From these capacitance values, we can roughly calculate the electrochemically effective surface area, and thus the turnover frequency per site $\left(0.030 \mathrm{~s}^{-1}\right.$ at $100 \mathrm{mV}$ and $0.219 \mathrm{~s}^{-1}$ at $150 \mathrm{mV}$, see Supplementary Table 3) by using a similar calculation method developed by Jaramillo et al. ${ }^{32}$ (Supplementary Note 5). The turnover frequency values are larger than many values reported on $\mathrm{MoS}_{2}$-like catalysts, and close to that of transition metal phosphide-based electrocatalysts (Supplementary Table 3). On the other hand, electrochemical impedance spectroscopy was carried out to examine the electrode kinetics under the catalytic HER-operating conditions (Fig. 3f). According to the Nyquist plots and data fitting to a simplified Randles circuit, our results clearly reveal that the charge-transfer resistance $\left(R_{\mathrm{ct}} \sim 0.5 \Omega\right)$ for the $\mathrm{MoS}_{2(1-x)} \mathrm{Se}_{2 x} / \mathrm{NiSe}_{2}$ hybrid is much smaller than that for pure $\mathrm{MoS}_{2} / \mathrm{NiSe}_{2}\left(R_{\mathrm{ct}} \sim 8 \Omega\right)$ or for porous $\mathrm{NiSe}_{2}$ foam alone $\left(R_{\mathrm{ct}} \sim 22 \Omega\right)$. In addition, all the catalysts have very small series resistances $\left(R_{\mathrm{s}} \sim 0.6-1.2 \Omega\right)$, suggesting high-quality electrical integration of the catalyst with the electrode.

Quantum mechanics calculations. To understand the improvement on the catalytic hydrogen evolution of the $\mathrm{MoS}_{2(1-x)} \mathrm{Se}_{2 x} / \mathrm{NiSe}_{2}$ hybrid catalyst, quantum mechanics calculations at the density functional theory (DFT) level (PBE-D3 flavor, see Supplementary Note 6) were performed to calculate the binding free energies of hydrogen on the Mo atom ${ }^{11,23}$. Although it was originally suggested that the edge $\mathrm{S}$ atom is the catalytic atom in hydrogen evolution on $\mathrm{MoS}_{2}$ (ref. 11), we find that $\mathrm{H}_{2}$ formation going through the Mo atom via the Heyrovsky reaction $^{33}$ has a lower barrier than the Heyrovsky and Volmer ${ }^{34}$ reaction on the $S$ atom. Therefore, we use a lower hydrogenbinding energy on the Mo atom as the indicator of a lower barrier in the Heyrovsky step. Since there are various exposed facets in our as-prepared $\mathrm{NiSe}_{2}$ foam (Supplementary Fig. 1), we modelled the reaction on the simple low-index (100), (110) and (111) surfaces of $\mathrm{NiSe}_{2}$. Molybdenum dichalcogenide with Se:S ratios of $0: 1,1: 1,1: 0$ are modelled, and, in the 1:1 case, the $S$ and Se alternate above and below the plane to avoid strain. As shown in Fig. $4 \mathrm{a}, \Delta G_{\mathrm{H}}{ }^{*}$ is $8.4 \mathrm{kcal} \mathrm{mol}^{-1}$ for hydrogen adsorbed on 
a
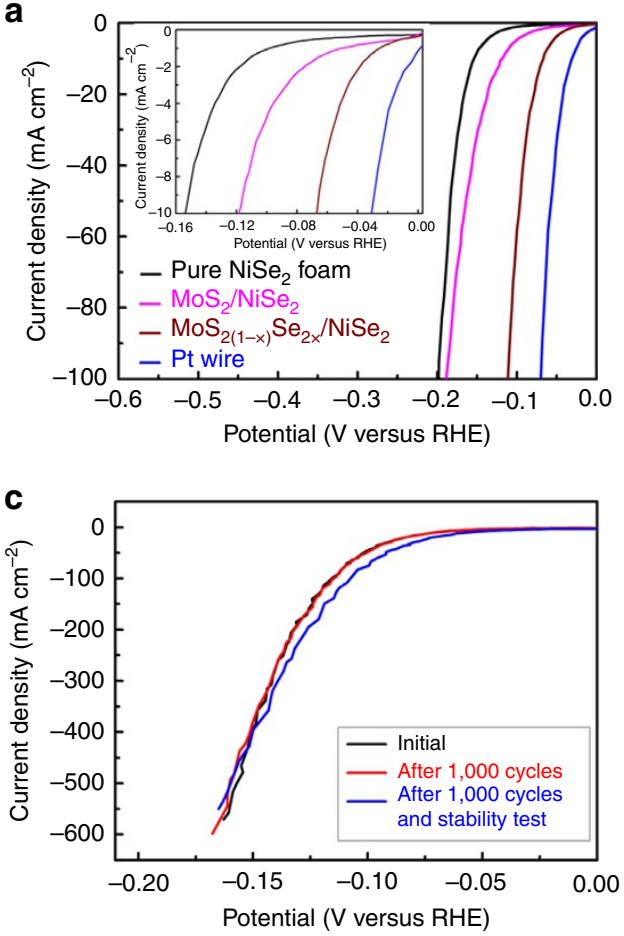

e

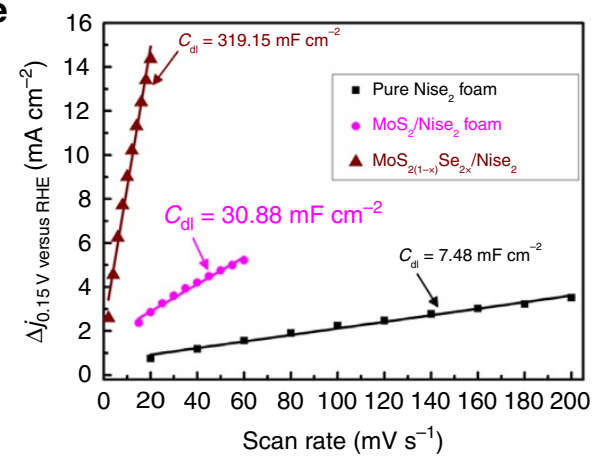

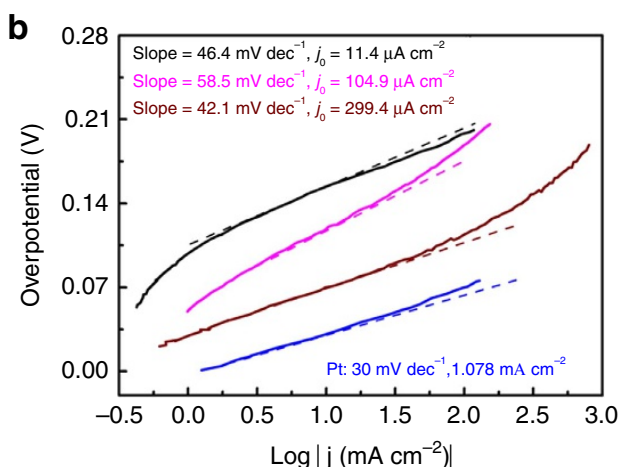

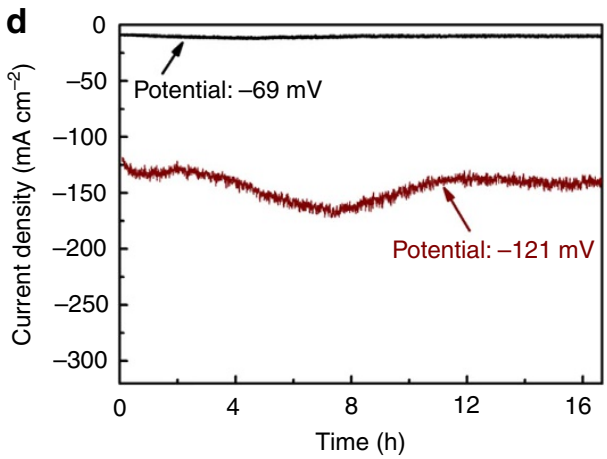

f

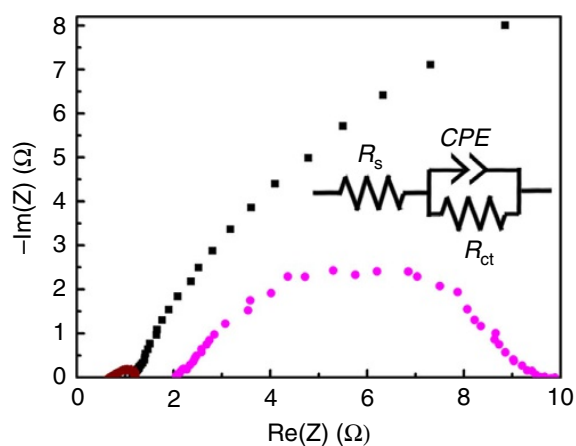

Figure 3 | Electrocatalytic performance of different catalysts. (a) The polarization curves recorded on $\mathrm{MoS}_{2(1-x)} \mathrm{Se}_{2 x} / \mathrm{NiSe}_{2}$ foam hybrid, MoS $/ \mathrm{NiSe}_{2}$ foam hybrid and pure $\mathrm{NiSe}_{2}$ foam electrodes compared with a Pt wire. (b) Tafel plots recorded on the catalysts in a. (c) Polarization curves showing negligible current density loss of ternary $\mathrm{MoS}_{2(1-x)} \mathrm{Se}_{2 x} / \mathrm{NiSe}_{2}$ hybrid electrodes initially, after 1,000 CV cycles and after the stability test. (d) Time dependence of current densities -10 and $-140 \mathrm{~mA} \mathrm{~cm}^{-2}$ recorded on the $\mathrm{MoS}_{2(1-x)} \mathrm{Se}_{2 x} / \mathrm{NiSe}_{2}$ hybrid electrode under given potentials of -69 and $-121 \mathrm{mV}$, respectively. (e) Plot showing the extraction of the $C_{d l}$ from different electrodes. (f) Electrochemical impedance spectroscopy (EIS) Nyquist plots of different electrocatalysts. The data were fit to the simplified Randles equivalent circuit shown in the inset. The loading of $\mathrm{MoS}_{2(1-x)} \mathrm{Se}_{2 x} \mathrm{catalyst}$ is $4.5 \mathrm{mg} \mathrm{cm}^{-2}$.

$\mathrm{MoS}_{2(1-x)} \mathrm{Se}_{2 x} / \mathrm{MoS}_{2(1-x)} \mathrm{Se}_{2 x}$, which is more reactive than $\mathrm{MoS}_{2} / \mathrm{MoS}_{2}$ with a $\Delta G_{\mathrm{H}}^{*}$ of $10.6 \mathrm{kcal} \mathrm{mol}^{-1}$, agreeing with the reported experimental results (Supplementary Figs 6 and 7) ${ }^{35,36}$. In contrast, once the $\mathrm{MoS}_{2(1-x)} \mathrm{Se}_{2 x}$ particles are hybridized with porous $\mathrm{NiSe}_{2}$ foam, the relevant $\Delta G_{\mathrm{H}}{ }^{*}$ on $\mathrm{MoS}_{2(1-x)} \mathrm{Se}_{2 x} / \mathrm{NiSe}_{2}$ (100) and $\mathrm{MoS}_{2(1-x)} \mathrm{Se}_{2 x} / \mathrm{NiSe}_{2}$ (110) are further decreased to 2.7 and $2.1 \mathrm{kcal} \mathrm{mol}^{-1}$, making these hybrid catalysts much more active than $\mathrm{MoS}_{2} / \mathrm{MoS}_{2}$ and $\mathrm{MoS}_{2(1-x)} \mathrm{Se}_{2 x} / \mathrm{MoS}_{2(1-x)} \mathrm{Se}_{2 x}$ in the HER process. To understand the reason for the improved reactivity of $\mathrm{MoS}_{2(1-x)} \mathrm{Se}_{2 x} / \mathrm{NiSe}_{2}$ hybrid catalysts, we examined the intermediate structures (Fig. 4b). When the $\mathrm{MoS}_{2(1-x)} \mathrm{Se}_{2 x}$ particles are placed on top of the $\mathrm{NiSe}_{2}$ substrate, they relax to form a chemically bonded hybrid on the (100) and (110) surfaces of $\mathrm{NiSe}_{2}$, while remaining unbonded from the (111) surface of $\mathrm{NiSe}_{2}$. Thus, DFT calculations corroborate that the $\mathrm{MoS}_{2(1-x)} \mathrm{Se}_{2 x} / \mathrm{NiSe}_{2}$ hybrid is a promising electrocatalyst (Fig. 4).

\section{Discussion}

In general, the as-prepared hybrid catalysts possess the merits of all the $\mathrm{MoS}_{2}$ catalysts that have ever been reported on improving the relevant catalytic performance ${ }^{18,19}$. Namely, the outstanding HER activity as well as good stability for ternary $\operatorname{MoS}_{2(1-x)} \mathrm{Se}_{2 x}$ particles/porous $\mathrm{NiSe}_{2}$ foam can be attributed to the synergistic effects from the dense catalytic edge sites at the $\operatorname{MoS}_{2(1-x)} \mathrm{Se}_{2 x}$ surface, good dispersion of the $\mathrm{MoS}_{2(1-x)} \mathrm{Se}_{2 x}$ particles on $\mathrm{NiSe}_{2}$ foam, good electrical contact and chemical bonding between $\mathrm{MoS}_{2(1-x)} \mathrm{Se}_{2 x}$ and $\mathrm{NiSe}_{2}$ catalysts, and 3D porous structures of HER-active $\mathrm{NiSe}_{2}$ foam: first, similar to $\mathrm{MoS}_{2}$, the catalytic property of $\mathrm{MoS}_{2(1-x)} \mathrm{Se}_{2 x}$ is greatly related to the number of exposed edge sites ${ }^{11,37}$. Indeed, in our experiments, because of the porous structure and curved surface of as-grown $\mathrm{NiSe}_{2}$ foam, ternary $\mathrm{MoS}_{2(1-x)} \mathrm{Se}_{2 x}$ layers tend to exhibit vertical orientation on the $\mathrm{NiSe}_{2}$ surface as demonstrated using high-resolution TEM, indicating that abundant active edge sites 
a
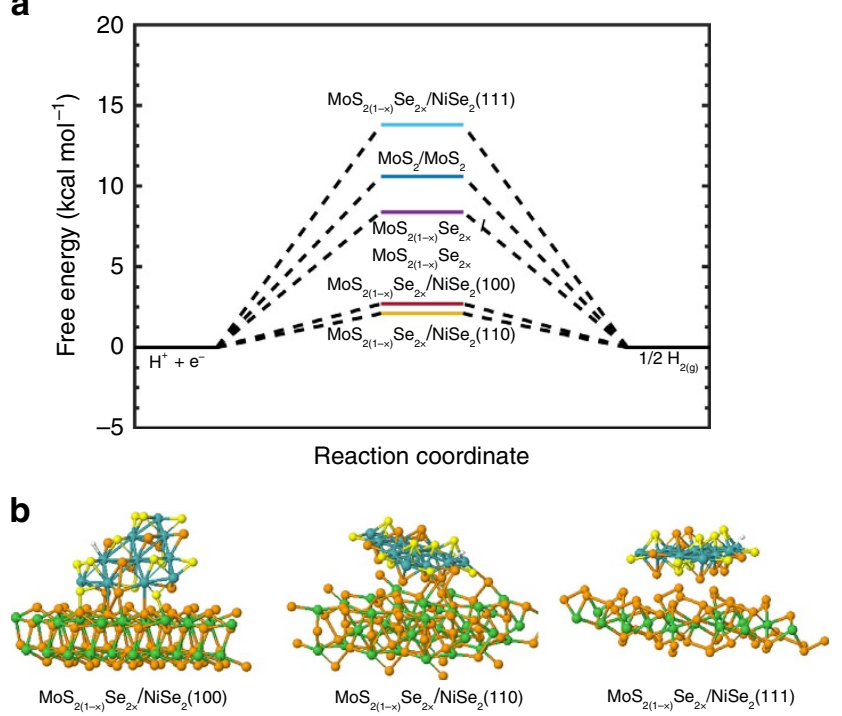

Figure 4 | Density functional theory calculations. (a) Calculated adsorption free energy diagram for hydrogen $\left(\mathrm{H}^{*}\right)$ adsorption at the equilibrium potential for $\mathrm{MoS}_{2(1-x)} \mathrm{Se}_{2 x} / \mathrm{NiSe}_{2}$ hybrid, binary $\mathrm{MoS}_{2}$ and ternary $\operatorname{MoS}_{2(1-x)} \mathrm{Se}_{2 x}$ catalysts. (b) Intermediate structures of hydrogenbound $\mathrm{MoS}_{2(1-x)} \mathrm{Se}_{2 x} / \mathrm{NiSe}_{2}$ (100), $\mathrm{MoS}_{2(1-x)} \mathrm{Se}_{2 x} / \mathrm{NiSe}_{2}$ (110) and $\mathrm{MoS}_{2(1-x)} \mathrm{Se}_{2 x} / \mathrm{NiSe}_{2}$ (111).

are exposed in these $\mathrm{MoS}_{2(1-x)} \mathrm{Se}_{2 x}$ particles ${ }^{27}$. Second, the electrical conductivity of $\mathrm{MoS}_{2(1-x)} \mathrm{Se}_{2 x}$ catalysts is another crucial factor to the electrocatalytic activity since the intrinsic conductivity is extremely low between two adjacent van der Waals bonded $\mathrm{S}-\mathrm{Mo}-\mathrm{S}$ layers ${ }^{14,16}$. In our case, the $\mathrm{MoS}_{2(1-x)} \mathrm{Se}_{2 x}$ layers are vertically oriented, which enhances the electron transfer from the electrode to the $\mathrm{MoS}_{2(1-x)} \mathrm{Se}_{2 x}$ layers. Furthermore, the underlying $\mathrm{NiSe}_{2}$ foam is metallic and is composed of lots of $3 \mathrm{D}$ porous structures that ensure rapid electron transport from the less-conducting $\mathrm{MoS}_{2(1-x)} \mathrm{Se}_{2 x}$ to the electrodes, and easy diffusion of the electrolyte into the active sites $^{18}$. Finally, the Gibbs free energy for hydrogen adsorption on $\mathrm{MoS}_{2(1-x)} \mathrm{Se}_{2 x}$ edges plays significant roles in this HER process, which is much lower compared with $\mathrm{MoS}_{2}$, leading to higher coverage of hydrogen adsorption at the active sites. Thus, by using this strategy, we can simultaneously engineer the catalysts with high surface area, 3D porous structures, good electrical conductivity and a large number of exposed active edge sites.

In summary, we propose and validate a simple and efficient strategy to synthesize a robust and stable self-standing hydrogenevolving catalyst by simply growing ternary $\operatorname{MoS}_{2(1-x)} \mathrm{Se}_{2 x}$ particles on $3 \mathrm{D}$ porous and metallic $\mathrm{NiSe}_{2}$ foam. According to our experimental results and quantum mechanics DFT calculation, these $\mathrm{MoS}_{2(1-x)} \mathrm{Se}_{2 x} / \mathrm{NiSe}_{2}$ hybrid catalysts exhibit an outstanding catalytic performance superior to that of the widely reported LTMD catalysts (especially $\mathrm{MoS}_{2}, \mathrm{WS}_{2}$ and so on) and first-row transition metal pyrites ( $\mathrm{CoSe}_{2}, \mathrm{CoS}_{2}$ and so on). Our catalysts are very effective in catalysing hydrogen production by integrating metal dichalcogenides and pyrites into $3 \mathrm{D}$ hybrid architectures that possess high surface area, porous structures, good electrical conductivity and abundant active edge sites, making it promising to realize large-scale water splitting.

\section{Methods}

Material synthesis. 3D porous $\mathrm{NiSe}_{2}$ foam was directly synthesized by thermal selenization of commercial $\mathrm{Ni}$ foam in a tube furnace. Then, the as-prepared $\mathrm{NiSe}_{2}$ foam was immersed in $\left(\mathrm{NH}_{4}\right)_{2} \mathrm{MoS}_{4}$ solution in dimethylformamide (DMF) solvent (5 wt\% $\left(\mathrm{NH}_{4}\right)_{2} \mathrm{MoS}_{4}$ in DMF) and dried on the hot plate, followed by thermolysis or second selenization at $500{ }^{\circ} \mathrm{C}$ in the tube furnace. The details are shown in the Supplementary Information.

Electrochemical measurements. The electrochemical measurements were conducted in a three-electrode setup with an electrochemical station (Gamry, Reference 600). The polarization curves were collected by linear sweep voltammetry with a scan rate of $0.5 \mathrm{mV} \mathrm{s}^{-1}$ in $82 \mathrm{ml}$ of $0.5 \mathrm{M} \mathrm{H}_{2} \mathrm{SO}_{4}$, so as to suppress the capacitive current due to the high surface area and high porosity of the porous samples ${ }^{38}$. A saturated calomel electrode was used as the reference electrode, a Pt wire ( $\mathrm{CH}$ Instruments Inc.) as the counter electrode and as-prepared hybrid catalysts as the self-supported working electrodes. During the electrochemical measurements, high-purity $\mathrm{N}_{2}$ gas was continually bubbled throughout the whole electrochemical measurement. Potentials versus RHE can be calculated compared with saturated calomel electrode by adding a value of $0.263 \mathrm{~V}$ after calibration. The electrochemical stability of the catalyst was evaluated by continuously cycling the catalyst for 1,000 times at a scan rate of $50 \mathrm{mV} \mathrm{s}^{-1}$. Chronoamperometry was performed under a given potential for the

$\mathrm{MoS}_{2(1-x)} \mathrm{Se}_{2 x} / \mathrm{NiSe}_{2}$ hybrid electrode. The electrochemical impedance spectroscopy test was carried out in the same device configuration at a potential of $-0.15 \mathrm{~V}$ versus RHE with the frequency ranging from $10 \mathrm{mHz}$ to $1 \mathrm{MHz}$ with a $10 \mathrm{mV}$ AC dither. All the potentials used here were referred to RHE.

Data availability. The data that support the findings of this study are available from the corresponding author upon request.

\section{References}

1. Bockris, J. O. M. The origin of ideas on a hydrogen economy and its solution to the decay of the environment. Int. J. Hydrogen Energy 27, 731-740 (2002).

2. Yang, Y. et al. A hybrid energy cell for self-powered water splitting. Energy Environ. Sci. 6, 2429-2434 (2013).

3. Gratzel, M. Photoelectrochemical cells. Nature 414, 338-344 (2001).

4. Cook, T. R. et al. Solar energy supply and storage for the legacy and nonlegacy worlds. Chem. Rev. 110, 6474-6502 (2010).

5. Liu, G. et al. Heteroatom-modulated switching of photocatalytic hydrogen and oxygen evolution preferences of anatase $\mathrm{TiO}_{2}$ microspheres. Adv. Funct. Mater. 22, 3233-3238 (2012).

6. Trasatti, S. Electrocatalysisof hydrogen evolution: progress in cathode activation. Adv. Electrochem. Sci. Eng. 2, 1-85 (1992).

7. Lu, Y. C., Gasteiger, H. A. \& Shao-Horn, Y. Catalytic activity trends of oxygen reduction reaction for nonaqueous Li-air batteries. J. Am. Chem. Soc. 133, 19048-19051 (2011).

8. Tang, W. et al. Self-powered water splitting using flowing kinetic energy. Adv. Mater. 27, 272-276 (2015).

9. Faber, M. S. \& Jin, S. Earth-abundant inorganic electrocatalysts and their nanostructures for energy conversion applications. Energy Environ. Sci. 7 3519-3542 (2014).

10. Morales-Guio, C. G., Stern, L. A. \& Hu, X. L. Nanostructured hydrotreating catalysts for electrochemical hydrogen evolution. Chem. Soc. Rev. 43, 6555-6569 (2014).

11. Hinnemann, B. et al. Biomimetic hydrogen evolution: $\mathrm{MoS}_{2}$ nanoparticles as catalyst for hydrogen evolution. J. Am. Chem. Soc. 127, 5308-5309 (2005).

12. Gao, M. R., Xu, Y. F., Jiang, J. \& Yu, S. H. Nanostructured metal chalcogenides: synthesis, modification, and applications in energy conversion and storage devices. Chem. Soc. Rev. 42, 2986-3017 (2013).

13. Karunadasa, H. I. et al. A molecular $\mathrm{MoS}_{2}$ edge site mimic for catalytic hydrogen generation. Science 335, 698-702 (2012).

14. Lukowski, M. A. et al. Enhanced hydrogen evolution catalysis from chemically exfoliated metallic $\mathrm{MoS}_{2}$ nanosheets. J. Am. Chem. Soc. 135, 10274-10277 (2013).

15. Lukowski, M. A. et al. Highly active hydrogen evolution catalysis from metallic $\mathrm{WS}_{2}$ nanosheets. Energy Environ. Sci. 7, 2608-2613 (2014).

16. Xie, J. F. et al. Controllable disorder engineering in oxygen- incorporated $\mathrm{MoS}_{2}$ ultrathin nanosheets for efficient hydrogen evolution. J. Am. Chem. Soc. 135, 17881-17888 (2013).

17. Li, Y. et al. $\mathrm{MoS}_{2}$ nanoparticles grown on graphene: an advanced catalyst for the hydrogen evolution reaction. J. Am. Chem. Soc. 133, 7296-7299 (2011).

18. Li, D. J. et al. Molybdenum sulfide/N-doped CNT forest hybrid catalysts for high- performance hydrogen evolution reaction. Nano Lett. 14, 1228-1233 (2014).

19. Kibsgaard, J., Chen, Z. B., Reinecke, B. N. \& Jaramillo, T. F. Engineering the surface structure of $\mathrm{MoS}_{2}$ to preferentially expose active edge sites for electrocatalysis. Nat. Mater. 11, 963-969 (2012).

20. Xu, K. et al. Component-controllable $\mathrm{WS}_{2(1-\mathrm{x})} \mathrm{Se}_{2 \mathrm{x}}$ nanotubes for efficient hydrogen evolution reaction. ACS Nano 8, 8468-8476 (2014).

21. Gao, M. R. et al. An efficient molybdenum disulfide/cobalt diselenide hybrid catalyst for electrochemical hydrogen generation. Nat. Commun. 6, 5982 (2015). 
22. Wang, X. G., Kolen'ko, Y. V., Bao, X. Q., Kovnir, K. \& Liu, L. F. One-step synthesis of self-supported nickel phosphide nanosheet array cathodes for efficient electrocatalytic hydrogen generation. Angew. Chem. Int. Ed. 54, 8188-8192 (2015)

23. Cabán-Acevedo, M. et al. Efficient hydrogen evolution catalysis using ternary pyrite-type cobalt phosphosulphide. Nat. Mater. 14, 1245-1251 (2015).

24. Kong, D. S., Wang, H. T., Lu, Z. Y. \& Cui, Y. CoSe ${ }_{2}$ nanoparticles grown on carbon fiber paper: an efficient and stable electrocatalyst for hydrogen evolution reaction. J. Am. Chem. Soc. 136, 4897-4900 (2014).

25. Chen, Z. P. et al. Three-dimensional flexible and conductive interconnected graphene networks grown by chemical vapour deposition. Nat. Mater. 10 424-428 (2011).

26. Zhou, H. Q. et al. One-step synthesis of self-supported porous $\mathrm{NiSe}_{2} / \mathrm{Ni}$ hybrid foam: an efficient 3D electrode for hydrogen evolution reaction. Nano Energy 20, 29-36 (2016).

27. Wang, H. T. et al. $\mathrm{MoSe}_{2}$ and $\mathrm{WSe} \mathrm{S}_{2}$ nanofilms with vertically aligned molecular layers on curved and rough surfaces. Nano Lett. 13, 3426-3433 (2013).

28. Gong, Y. J. et al. Band gap engineering and layer-by-layer mapping of selenium-doped molybdenumd disulfide. Nano Lett. 14, 442-449 (2014).

29. Li, H. L. et al. Growth of alloy $\mathrm{MoS}_{2 \mathrm{x}} \mathrm{Se}_{2(1-\mathrm{x})}$ nanosheets with fully tunable chemical compositions and optical properties. J. Am. Chem. Soc. 136, 3756-3759 (2014)

30. Wang, F. M. et al. Enhanced electrochemical $\mathrm{H}_{2}$ evolution by few-layered metallic $\mathrm{WS}_{2(1-\mathrm{x})} \mathrm{Se}_{2 \mathrm{x}}$ nanoribbons. Adv. Funct. Mater. 25, 6077-6083 (2015).

31. Peng, S. J. et al. Cobalt sulfide nanosheet/ graphene/carbon nanotube nanocomposites as flexible electrodes for hydrogen evolution. Angew. Chem. Int. Ed. 126, 12802-12807 (2014)

32. Benck, J. D., Chen, Z. B., Kuritzky, L. Y., Forman, A. J. \& Jaramillo, T. F. Amorphous molybdenum sulfide catalysts for electrochemical hydrogen production: insights into the origin of their catalytic activity. ACS Catal. 2 , 1916-1923 (2012).

33. Tsai, C., Chan, K., Nørskov, J. K. \& Abild-Pedersen, F. Theoretical insights into the hydrogen evolution activity of layered transition metal dichalcogenides. Surface Sci. 640, 133-140 (2015).

34. Huang, Y. F., Nielsen, R. J., Goddard III, W. A. \& Soriaga, M. P. The reaction mechanism with free energy barriers for electrochemical dihydrogen evolution on $\mathrm{MoS}_{2}$. J. Am. Chem. Soc. 137, 6692-6698 (2015).

35. Gong, Q. F. et al. Ultrathin $\mathrm{MoS}_{2(1-\mathrm{x})} \mathrm{Se}_{2 \mathrm{x}}$ alloy nanoflakes for electrocatalytic hydrogen evolution reaction. ACS Catal. 5, 2213-2219 (2015).

36. Kiran, V., Mukherjee, D., Jenjeti, R. N. \& Sampath, S. Active guests in the $\mathrm{MoS}_{2} / \mathrm{MoSe}_{2}$ host lattice: efficient hydrogen evolution using few-layer alloys of $\mathrm{MoS}_{2(1-\mathrm{x})} \mathrm{Se}_{2 \mathrm{x}}$. Nanoscale 6, 12856-12863 (2014).

37. Jaramillo, T. F. et al. Identification of active edge sites for electrochemical $\mathrm{H}_{2}$ evolution from $\mathrm{MoS}_{2}$ nanocatalysts. Science 317, 100-102 (2007).
38. Lu, Q. et al. Highly porous non-precious bimetallic electrocatalysts for efficient hydrogen evolution. Nat. Commun. 6, 6567 (2015).

\section{Acknowledgements}

This project was partially supported by US Defense Threatening Reduction Agency (DTRA) under grant FA 7000-13-1-0001, and the computational work was supported through the Office of Science of US Department of Energy under Award No. DE-SC0004993. J.M.B. acknowledges the support from the National Science Foundation (CAREER Award ECCS-1240510) and the Robert A. Welch Foundation (E-1728).

\section{Author contributions}

Z.R. guided the project and discussed the experimental results. H.Z. conceived, designed and performed the experiments (SEM, Raman, XPS and HER tests) and analysed the data. F.Y. conducted catalyst synthesis by CVD method and helped to collect the HER data. Y.H., R.J.N. and W.A.G. carried out the first-principles calculations. J.S. performed TEM characterizations. R.H. carried out XRD characterization. S.C. contributed to the result discussion, device design and measurements. Z.Z. and J.B. measured the Faradaic efficiency. H.Z., S.C. and Z.R. wrote the paper. All the authors discussed the results and revised the paper.

\section{Additional information}

Supplementary Information accompanies this paper at http://www.nature.com/ naturecommunications

Competing financial interests: The authors declare no competing financial interests.

Reprints and permission information is available online at http://npg.nature.com/ reprintsandpermissions/

How to cite this article: Zhou, H. et al. Efficient hydrogen evolution by ternary molybdenum sulfoselenide particles on self-standing porous nickel diselenide foam. Nat. Commun. 7:12765 doi: 10.1038/ncomms12765 (2016).

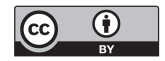

This work is licensed under a Creative Commons Attribution 4.0 International License. The images or other third party material in this article are included in the article's Creative Commons license, unless indicated otherwise in the credit line; if the material is not included under the Creative Commons license, users will need to obtain permission from the license holder to reproduce the material. To view a copy of this license, visit http://creativecommons.org/licenses/by/4.0/

(C) The Author(s) 2016 